\title{
Integrated Optical Components Utilizing Long-Range Surface Plasmon Polaritons
}

Boltasseva, Alexandra; Nikolajsen, Thomas; Leosson, Kristjan; Kjær, Kasper; Larsen, Morten S.; Bozhevolnyi, Sergey I.

Published in:

Journal of Lightwave Technology

Link to article, DOI:

10.1109/JLT.2004.835749(410)23

Publication date:

2005

Document Version

Publisher's PDF, also known as Version of record

Link back to DTU Orbit

Citation $(A P A)$ :

Boltasseva, A., Nikolajsen, T., Leosson, K., Kjær, K., Larsen, M. S., \& Bozhevolnyi, S. I. (2005). Integrated Optical Components Utilizing Long-Range Surface Plasmon Polaritons. Journal of Lightwave Technology, 23(1), 413-422. https://doi.org/10.1109/JLT.2004.835749(410)23

\section{General rights}

Copyright and moral rights for the publications made accessible in the public portal are retained by the authors and/or other copyright owners and it is a condition of accessing publications that users recognise and abide by the legal requirements associated with these rights.

- Users may download and print one copy of any publication from the public portal for the purpose of private study or research.

- You may not further distribute the material or use it for any profit-making activity or commercial gain

- You may freely distribute the URL identifying the publication in the public portal 


\title{
Integrated Optical Components Utilizing Long-Range Surface Plasmon Polaritons
}

\author{
Alexandra Boltasseva, Thomas Nikolajsen, Kristjan Leosson, Kasper Kjaer, Morten S. Larsen, and
} Sergey I. Bozhevolnyi

\begin{abstract}
New optical waveguide technology for integrated optics, based on propagation of long-range surface plasmon polaritons (LR-SPPs) along metal stripes embedded in dielectric, is presented. Guiding and routing of electromagnetic radiation along nanometer-thin and micrometer-wide gold stripes embedded in polymer via excitation of LR-SPPs is investigated in the wavelength range of 1250-1650 $\mathrm{nm}$. LR-SPP guiding properties, such as the propagation loss and mode-field diameter, are investigated for different stripe widths and thicknesses. A propagation loss of $\sim 6 \mathrm{~dB} / \mathrm{cm}$, a coupling loss of $\sim 0.5 \mathrm{~dB}$ (per facet), and a bend loss of $\sim 5 \mathrm{~dB}$ for a bend radius of $15 \mathrm{~mm}$ are evaluated for $15-\mathrm{nm}$-thick and $8-\mu \mathrm{m}$-wide stripes at the wavelength of $1550 \mathrm{~nm}$. LR-SPP-based 3-dB power $Y$-splitters, multimode interference waveguides, and directional couplers are demonstrated and investigated. At $1570 \mathrm{~nm}$, coupling lengths of 1.9 and $0.8 \mathrm{~mm}$ are found for directional couplers with, respectively, 4- and 0 - $\mu \mathrm{m}$-separated waveguides formed by $15-\mathrm{nm}$-thick and 8 - $\mu \mathrm{m}$-wide gold stripes. LR-SPP-based waveguides and waveguide components are modeled using the effective-refractive-index method, and good agreement with experimental results is obtained.
\end{abstract}

Index Terms-Directional couplers (DCs), integrated optics, surface waves, waveguide components.

\section{INTRODUCTION}

$\mathbf{I}$ NTEGRATED optical circuits have been intensively developing over the last 30 years with the purpose of reducing the number of electrooptic interconnections and realizing various functionalities, such as optical routing, splitting, combining, and filtering of optical signals. Currently, several integrated optical technologies are developed with different technological platforms and material systems, including III-V semiconductors, silica, silica-on-silicon, and silicon oxynitride with processing based on standard photolithography [1]-[3], lithium niobate crystals modified with the titanium diffusion [4], as well as technologies based on sol-gel [5], polymers [6], and organo-mineral materials [7]. All existing technologies have their advantages and drawbacks but all have proven to be of preference for different applications. One of the most important characteristics of a technology is its price. Today, low-cost processes are thought to become the winners of future integrated-optic technology competition. A new approach, which

Manuscript received May 21, 2004.

A. Boltasseva is with Research Center COM, Technical University of Denmark, DK-2800 Kongens Lyngby, Denmark (e-mail: aeb@com.dtu.dk).

T. Nikolajsen, K. Leosson, K. Kjaer, and M. S. Larsen are with Micro Managed Photons A/S, DK-3520 Farum, Denmark.

S. I. Bozhevolnyi is with Micro Managed Photons A/S, DK-3520 Farum, Denmark, and Department of Physics and also with Nanotechnology, Aalborg University, DK-9220 Aalborg Øst, Denmark.

Digital Object Identifier 10.1109/JLT.2004.835749 suggests to employ long-range surface plasmon polaritons (LR-SPPs) for the guiding of light along thin metal stripes embedded in dielectric, e.g., polymer, seems to meet low-cost simplicity of fabrication, flexibility, as well as performance requirements.

Surface plasmon polaritons (SPPs) represent quasi-two-dimensional electromagnetic excitations propagating along a dielectric-metal interface [8]. The SPP field components decay exponentially into both neighboring media from their maxima at the interfaces. Rather tight field intensity confinement to the metal surface (typically on the order of or smaller than the wavelength in the corresponding media) makes SPPs very sensitive to surface irregularities so that the SPP scattering has been long considered as a tool for surface analysis [8]. Since then, many fundamental properties of SPPs propagating along the interface between metal and dielectric have been studied, and many interesting findings and possible applications have been reported [8]-[17]. Recent progress in the design and fabrication of highly integrated optical circuits has paved the way for exploitation of SPPs for radiation guiding along straight and sharply bent channels in (periodically) corrugated regions [13]-[15] and along narrow metal stripes [16], [17]. Due to the relatively small propagation length $(\sim 30 \mu \mathrm{m}$ in visible and $\sim 300 \mu \mathrm{m}$ in the near-infrared wavelength range for a silver-air interface [8], SPPs are considered to be somewhat limited in their applications. However, by changing a metal-dielectric interface to a symmetrical structure of a thin metal film embedded in dielectric, one can significantly decrease the SPP propagation loss [8]. In this symmetrical structure, two identical SPPs associated with the two (upper and lower) metal-dielectric interfaces become coupled, forming two modes: symmetrical and asymmetrical (with respect to the orientation of the main electric field component). It is the symmetrical mode that is of great interest and importance from the point of view of potential applications for two reasons. First, the attenuation of the symmetrical mode, also called LR-SPP, decreases drastically with the decrease of the film thickness, leading to an increase in the propagation length [18]. At the same time, for sufficiently thin metal films, the LR-SPP field components extend over several micrometers into the cladding via two identical evanescent tails, facilitating the optical excitation, because the mode size is now close to that of the standard SMF. The end-fire excitation of LR-SPPs was first proposed by Burke et al. [19] and realized by Charbonneau et al., who observed experimentally LR-SPP propagation along thin gold stripes of finite width embedded in silica glass [19], [20]. Quite recently, efficient LR-SPP excitation and guiding (at telecom wavelengths) along 10-nm-thin gold stripes embedded 
in polymer was realized demonstrating the coupling loss of $\sim 0.5$ $\mathrm{dB}$ and propagation loss of $\sim 6-8 \mathrm{~dB} / \mathrm{cm}$ [21].

Once low-loss LR-SPP propagation along straight stripes has been demonstrated, it seems natural to start investigating bends, splitters, and other waveguide components based on LR-SPP stripe waveguides. Bends, which are used for connecting waveguides offset with respect to each other, are very important in integrated optical circuits, because it is often required to properly adjust the separation between adjacent input/output waveguides with respect to that between in-/out-coupled (pigtailed) fibers. Over the years, wave propagation in bent waveguides has been studied extensively both theoretically and experimentally for different bend types, and different bend performances (including the smallest bend radius and bend loss) have been reported [22]-[24]. In integrated optical circuits, bends are usually included as parts of larger basic building blocks, such as Y-splitters and DCs. These components have generated a lot of interest due to their numerous applications for switching, modulation, wavelength demultiplexing, and power splitting [25]. In recent years, there has also been a growing interest in multimode interference (MMI) waveguide components that offer flexible and reconfigurable functionality used for signal routing and coupling devices [26]. Optical devices based on MMI effects are incorporated in modern phase diversity networks, Mach-Zehnder switches and modulators, balanced coherent receivers, ring lasers, and other integrated optical circuits and components.

The present paper reports the design, modeling, fabrication, and optical characterization of polymer-based LR-SPP stripe waveguides and waveguide components at telecommunication wavelengths. The guiding and routing of light along thin gold stripes embedded in polymer via excitation of LR-SPPs for different thicknesses and widths of stripes are investigated.

The paper is organized as follows. In Section II, modeling of LR-SPP propagation along thin metal films and finite-width stripes is considered by numerical solving of the dispersion relation. Propagation loss is calculated for different thicknesses of metal film and different properties of cladding layers (thickness and refractive index). Using the effective-refractive-index method, the dependencies of the propagation loss and lateral mode-field diameter (MFD) on the stripe width are simulated. Section III is devoted to experimental results obtained with the LR-SPP stripe waveguides and waveguide components. First, the fabrication procedure and experimental arrangements are described. Second, experimental values for the propagation loss for different stripe thicknesses are presented along with the mode profiles measured for different stripe widths. LR-SPP stripe waveguide bending and splitting is investigated experimentally for $8-\mu \mathrm{m}$-wide stripes. Lateral field profiles for optical MMI devices based on wide-stripe waveguides are measured for different waveguide lengths and compared with the results of modeling. Routing of light using DCs based on LR-SPP stripe waveguides is demonstrated for different stripe widths and separations. Coupling lengths are determined for DCs with different parameters showing good agreement with the values estimated using the effective-index method. Finally, the results are summarized and conclusions are offered in Section IV.

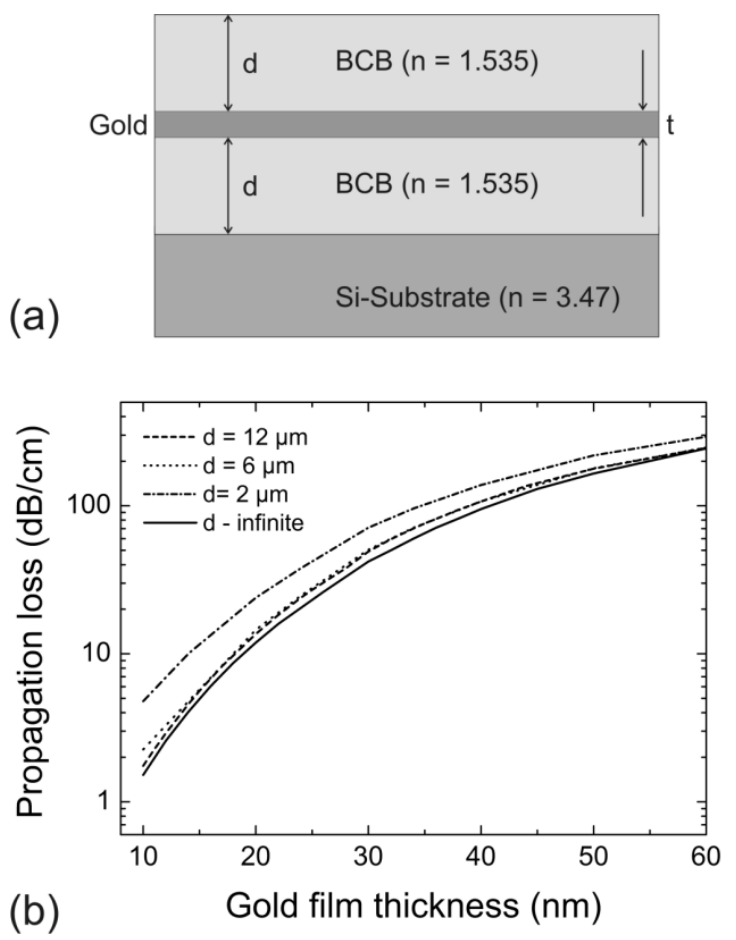

Fig. 1. (a) Symmetrical geometry of an infinitely wide metal film of variable thickness $t$ surrounded by two identical polymer $(n=1.535)$ layers of variable thickness $d$. The structure is placed on a silicon substrate $(n=3.47)$. (b) Dependence of the LR-SPP propagation loss on the gold film thickness at the wavelength of $1550 \mathrm{~nm}$ for different thicknesses of polymer cladding layers. The vertical-mode profiles for the 20 -nm-thick gold film are shown in the inset for two different cladding thicknesses.

\section{LR-SPP STRIPE WAVEGUIDES: EFFECTIVE-INDEX APPROACH}

\section{A. LR-SPPs Supported by a Thin Metal Film of Infinite Width}

Characteristics of SPP waves propagating along thin metal films embedded in dielectric were first considered by Sarid, who showed that the imaginary part of a propagation constant of the symmetrical mode, i.e., the LR-SPP mode, goes to zero as the metal film thickness decreases, implying the possibility of achieving very long propagation distances [18]. Properties of the LR-SPP mode supported by a thin metal film of infinite width can be analyzed by solving a dispersion relation [19] for confined electromagnetic waves obtained from the boundary condition for the tangential field components. In our work, we studied the LR-SPP characteristics for infinitely wide stripes using a one-dimensional mode solver by Kymata Software.

The first symmetrical geometry considered is shown in Fig. 1(a). A metal film of variable thickness $t$ is surrounded by two identical dielectric layers characterized by the refractive index $n=1.535$, corresponding to the refractive index of BCB (Benzocyclobutene) polymer at the light wavelength of $1.55 \mu \mathrm{m}$, and variable thickness $d$. The structure is placed on a silicon substrate with the refractive index of 3.47. The metal in our analysis is gold with the complex refractive index $n=0.55+11.5 i$ (this value is in fact also close to that of silver at $1.55 \mu \mathrm{m})$.

We analyzed the LR-SPP propagation loss at the wavelength of $1.55 \mu \mathrm{m}$ for different thicknesses of metal film and $\mathrm{BCB}$ 

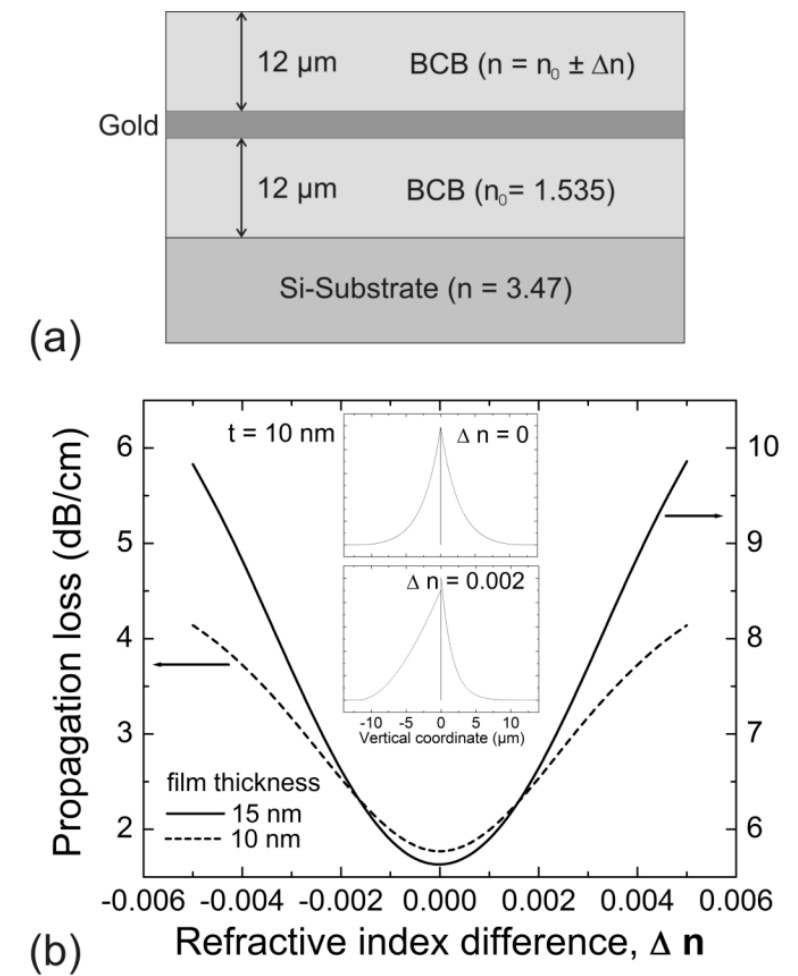

Fig. 2. (a) Same geometry as in Fig. 1(a) for a polymer cladding thickness of $12 \mu \mathrm{m}$ only with the variable refractive index of the top polymer cladding. (b) Dependence of the LR-SPP propagation loss on the refractive-index difference between two polymer claddings at the wavelength of $1550 \mathrm{~nm}$ for 10 - and 15 -nm-thick gold film. The vertical-mode profiles for the 10-nm-thick gold film are shown in the inset for 0 and 0.002 differences between cladding indexes.

cladding [Fig. 1(b)]. For infinite polymer cladding, the propagation loss was found to increase monotonically when increasing film thickness from $\sim 1.5 \mathrm{~dB} / \mathrm{cm}$ (for a 10-nm-thick gold film) to $\sim 250 \mathrm{~dB} / \mathrm{cm}$ (for the film thickness of $60 \mathrm{~nm}$ ). It should be emphasized that in order to support LR-SPP propagation, one should ensure a symmetrical structure. This means that two polymer layers should have the same refractive index and be sufficiently thick so that the LR-SPP field is located inside the polymer and does not penetrate into the silicon substrate or air. The LR-SPP mode profile in depth (perpendicular to the sample surface) is mainly determined by the metal thickness and reflects how tight the LR-SPP is bound to the metal. Here, we should mention that, in turn, the cladding (polymer) thickness can be used to tune the LR-SPP depth profile [21] as demonstrated in the inset of Fig. 1(b). For the gold thickness of $20 \mathrm{~nm}$, the breadth of the LR-SPP depth profile changes from $\sim 10 \mu \mathrm{m}$ for a $12-\mu \mathrm{m}$-thick cladding to $\sim 4 \mu \mathrm{m}$ for the polymer thickness of $2 \mu \mathrm{m}$. However, besides the control of the LR-SPP depth profile, the decrease in the cladding thickness increases the propagation loss. For example, for a 10-nm-thick metal film, reducing polymer thickness to $2 \mu \mathrm{m}$ will change the LR-SPP propagation loss from $\sim 1.5$ to $\sim 5 \mathrm{~dB} / \mathrm{cm}$ [Fig. 1(b)].

To study the influence of asymmetry in the cladding indexes on LR-SPP properties, we analyzed the same geometry as in Fig. 1(a) for the cladding thickness of $12 \mu \mathrm{m}$ and with the variable refractive index of the top cladding [Fig. 2(a)]. The dependence of the LR-SPP propagation loss on the refractive-index difference between top and bottom cladding layers is shown in

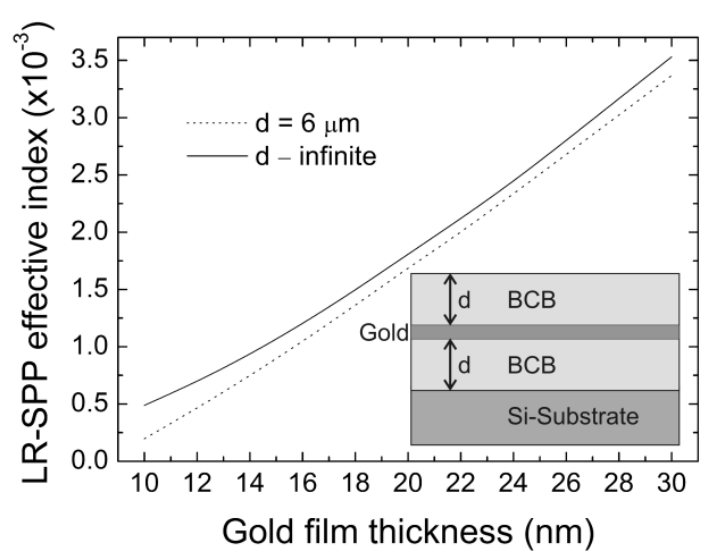

Fig. 3. Dependence of the LR-SPP effective refractive index on the gold film thickness for the infinite and 6- $\mu \mathrm{m}$-thick polymer cladding.

Fig. 2(b) for gold thicknesses of 10 and $15 \mathrm{~nm}$. For example, for a 10-nm-thick film, the LR-SPP mode was found to have the propagation loss increasing from $1.7 \mathrm{~dB} / \mathrm{cm}$ (for the symmetrical structure) to $\sim 4 \mathrm{~dB} / \mathrm{cm}$ (for the refractive index difference of \pm 0.006 ). The increase in the propagation loss with the increasing asymmetry is accompanied with the change from a symmetrical LR-SPP mode depth profile to an asymmetrical one [inset of Fig. 2(b)]. A further increase of the refractive-index difference (more than \pm 0.006 ) will create a conventional slab waveguide formed by a polymer layer with a higher refractive index surrounded by two media with lower refractive indexes, resulting in the propagating mode of the slab waveguide instead of the LR-SPP mode.

The dependence of the LR-SPP normalized effective refractive index $b$ on the gold film thickness is presented in Fig. 3 with the normalized index $b$ being conveniently determined as

$$
b=\frac{\beta-k_{0} n_{\mathrm{cl}}}{k_{0} n_{\mathrm{cl}}}=\frac{N_{\mathrm{eff}}-n_{\mathrm{cl}}}{n_{\mathrm{cl}}}
$$

where $\beta$ is the LR-SPP propagation constant, $\lambda$ is the light wavelength $(1.55 \mu \mathrm{m}), n_{\mathrm{cl}}$ is the refractive index of the cladding (1.535), and $N_{\mathrm{eff}}$ is the mode effective refractive index.

\section{B. LR-SPPs Supported by a Thin Metal Film of Finite Width}

The properties of LR-SPP modes guided by a waveguide structure composed of a thin lossy metal film of finite width, surrounded by dielectric, were for the first time studied theoretically by Berini [27]. In our simple qualitative analysis, the characteristics of the LR-SPP mode propagating in a stripe metal waveguide of finite width were found by using the effective-refractive-index method, which is considered to be reasonably accurate for waveguide modes being far from cutoff [28] and found to give fairly good predictions for the behavior of LR-SPP stripe waveguides. The geometry that we considered is shown in Fig. 4(a). A metal strip of variable thickness $t$ and width $w$ is surrounded by polymer characterized by the refractive index $n$, and the whole structure is placed on a silicon substrate.

In the first step, the structure with an infinitely wide metal film is analyzed resulting in the vertical LR-SPP mode profile and the effective index, which is used in the second step as the 
(a)
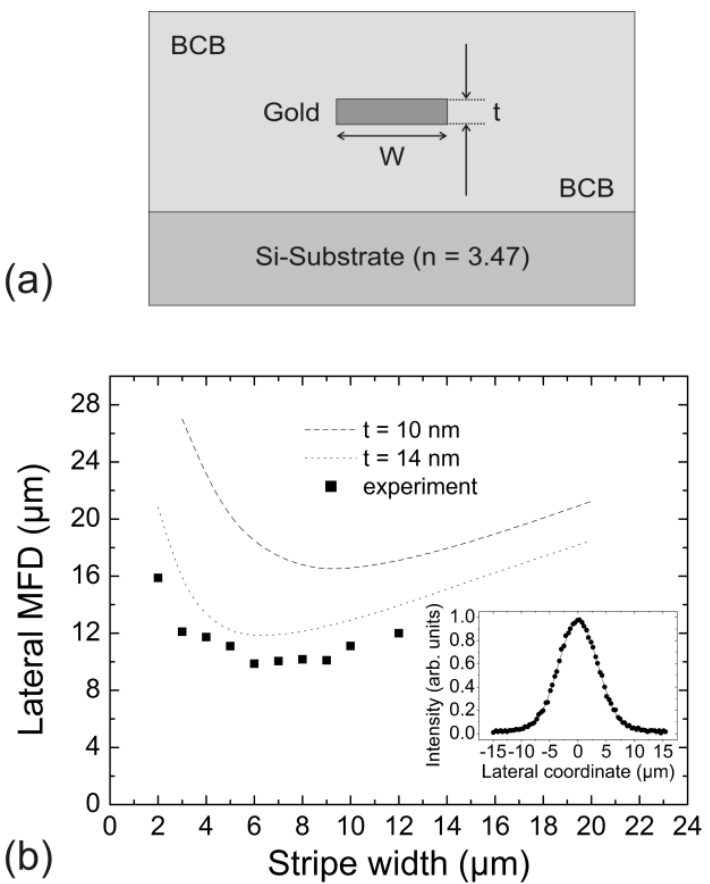

Fig. 4. (a) Geometry of a metal stripe of variable thickness $t$ and width $w$ surrounded by polymer $(n=1.535)$ layers. The structure is placed on a silicon substrate $(n=3.47$ ). (b) Dependence of the lateral LR-SPP MFD on the stripe width for gold film thicknesses of 10 and $14 \mathrm{~nm}$. Modeling performed using the effective-index approach. Dots represent the values measured for 15-nm-thick stripes sandwiched between $15-\mu \mathrm{m}$-thick polymer cladding layers. The inset shows an example of the lateral intensity profile fitted to a Gaussian distribution.

refractive index of a core in the slab waveguide configuration (the core thickness is considered equal to the stripe width). The waveguide analysis at the second step provides us with the lateral mode profile (parallel to the sample surface) as well as the corrected value for the mode effective refractive index and propagation loss. The lateral LR-SPP MFD is shown in Fig. 4(b) as a function of the stripe width for gold film thicknesses of 10 and $14 \mathrm{~nm}$. A typical behavior of the lateral LR-SPP MFD was found first to decrease following the decrease in the stripe width and then to increase again, emonstrating a poor light confinement by narrow stripes [27].

The LR-SPP mode effective index,together with the propagation loss as a function of the waveguide width for a 10-nm-thick stripe, s shown in Fig. 5. The simulations indicated that, for the stripe thickness of $10 \mathrm{~nm}$, the multimode regime sets in for stripes wider than $20 \mu \mathrm{m}$ [Fig. 5(a)] This feature was used to design MMI waveguides (Section III-F). The propagation loss was found to decrease with the stripe width (a similar trend was also predicted by Berini [27]) implying the possibility to reach very low propagation loss. For example, the propagation loss below $1 \mathrm{~dB} / \mathrm{cm}$ can be achieved for a $10-\mathrm{nm}$-thick stripe by reducing its widths below $5 \mu \mathrm{m}$ [Fig. 5(b)] .

\section{EXPERIMENTAL RESULTS}

\section{A. Sample Fabrication}

To fabricate LR-SPP stripe waveguides, a silicon substrate $\left(4^{\prime \prime}\right.$ or $\left.6^{\prime \prime}\right)$ was first spin-coated with a layer of polymer BCB with a thickness of $13-15 \mu \mathrm{m}$ and then with a layer of ultraviolet (UV) resist. Stripe waveguides and waveguide devices were

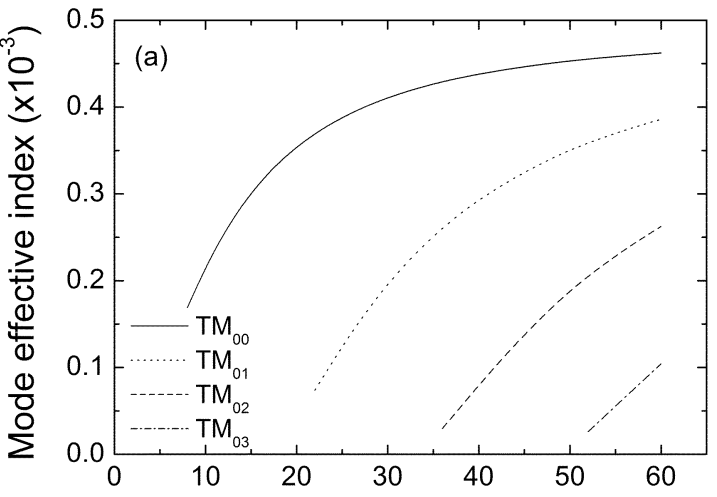

(a)

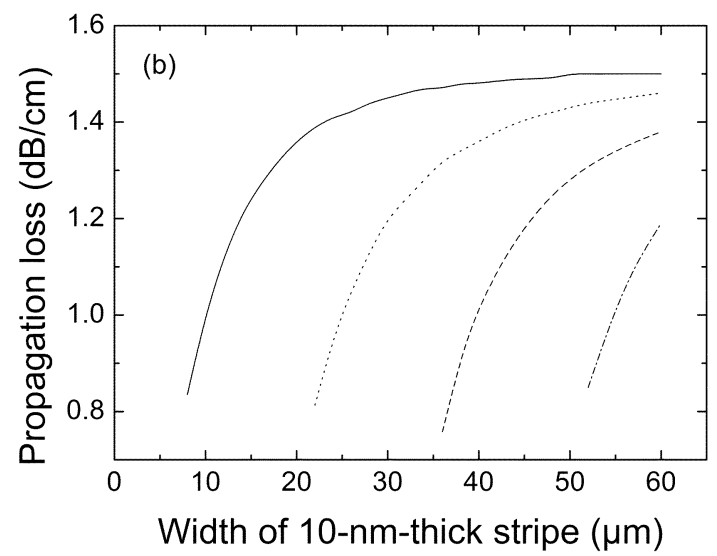

(b)

Fig. 5. (a) SPP mode effective index (b) together with the propagation loss as a function of the waveguide width for a 10-nm-thick stripe. Modeling performed using the effective-index approach.

patterned using standard UV lithography, gold deposition, and liftoff. As a final fabrication step, the spin-coating with the top cladding, comprising another 13- to 15 - $\mu$ m-thick BCB layer, was performed. Since the symmetry of the structure is very important for the LR-SPP properties (propagation loss, MFD), we controlled carefully that the cladding layers had the same refractive index and were thick enough to accommodate the electromagnetic (EM) field of the LR-SPP. This was guaranteed by applying the same polymer for the top and bottom claddings and using identical spinning and curing conditions. After the final polymer curing the wafer was cut into individual samples containing different waveguide devices.

Fabricated samples included straight stripe waveguides of different widths (from 2-12 $\mu \mathrm{m}$ ), S-bends of different lengths with $200-\mu \mathrm{m}$ offset, Y-splitters, 40- and 60- $\mu \mathrm{m}$-wide MMI waveguides connected to injection single-mode (8- $\mu \mathrm{m}$-wide) waveguides positioned symmetrically with respect to the MMI center line, and DCs based on 8 - $\mu \mathrm{m}$-wide stripe waveguides.

\section{B. Experimental Arrangement}

For optical characterization of the LR-SPP stripe waveguides and waveguide devices, standard transmission measurements were performed. In order to excite the LR-SPP mode, end-fire coupling of light was performed using a tunable laser (1550 
or $1570 \mathrm{~nm}$ ) or a broad-band light source (two multiplexed edge-emitting-light-emitting diodes-1310 and $1550 \mathrm{~nm}$ ), together with a polarization controller, as a source. Light polarized perpendicular to the waveguide plane was launched into the LR-SPP waveguide via butt-coupling from a polarization-maintaining (PM) fiber with an MFD of $10.8 \mu \mathrm{m}$. To ensure that the polarization of light was orthogonal to the waveguide layer, angular adjustments of the PM fiber were performed. 1 $\mathrm{km}$ of coiled standard SMF was used as out-coupling fiber in order to strip off all light coupled into the fiber cladding. Index matching gel was used to decrease the reflection at the sample edges. The output signal was detected by a power meter (for measurements performed with the laser) or optical spectrum analyzer (for broad-band measurements).

The adjustment of the in- and out-coupling fibers with respect to the stripe waveguide was accomplished by maximizing the amount of light transmitted through a waveguide (active alignment). We should mention that almost all light coupled from the input fiber to the cladding was stripped away before reaching the end facet of the sample so that only the LR-SPP mode confined to a waveguide was observed at the output, making the alignments of the fibers quite easy. The output intensity distribution from a stripe waveguide was monitored via an infrared vidicon camera through $200^{\prime}$ magnification. The PM fiber output with the known MFD was used for the calibration of the mode profile measurement system.

\section{Straight Stripe Waveguides}

The propagation loss measurements were performed for 8 - $\mu \mathrm{m}$-wide straight stripe waveguides of different thicknesses (thickness of the deposited gold layer) from approximately 8.5 to $35 \mathrm{~nm}$. At a particular wavelength, the propagation loss was found as the slope of the linear fit to the experimental values of loss obtained for different lengths of the LR-SPP waveguide $(4,8$, and $14 \mathrm{~mm}$ for waveguide thicknesses up to $15 \mathrm{~nm}$, and 2,3 , and $4 \mathrm{~mm}$ for thicknesses up to $35 \mathrm{~nm}$ ) (cutback method). This linear fitting technique allowed us to estimate the coupling loss from the intersection point on the loss axis corresponding to zero length of the waveguide. The value of the coupling loss for a 15-nm-thick stripe waveguide varied from approximately $0.5 \mathrm{~dB}$ per facet for a $10-\mu \mathrm{m}$-wide waveguide to $\sim 1.5 \mathrm{~dB}$ for a 4 - $\mu \mathrm{m}$-wide stripe. Fig. 6 shows the experimental results for the propagation loss at $1550 \mathrm{~nm}$, together with the LR-SPP propagation loss curve calculated for infinitely wide stripes. Good agreement between experimental and calculated values, observed for waveguide thicknesses higher than $15 \mathrm{~nm}$, clearly indicates that, for thick stripes, the internal damping in metal (ohmic loss) is dominating. For thin stripes, higher values of experimentally obtained propagation loss compared with the calculated values can be explained by the presence of other loss mechanisms such as the scattering by inhomogeneities in the gold structure, at the waveguide edges, and scattering and absorption in the polymer. By eliminating the described loss mechanisms, one should achieve the loss limit set by the internal damping in metal, which is $\sim 1.5 \mathrm{~dB} / \mathrm{cm}$ for a 10-nm-thick infinitely wide stripe and decreases with the stripe width [27]. Further reduction of the stripe thickness (less than $10 \mathrm{~nm}$ ) will hardly lead to a significant decrease in

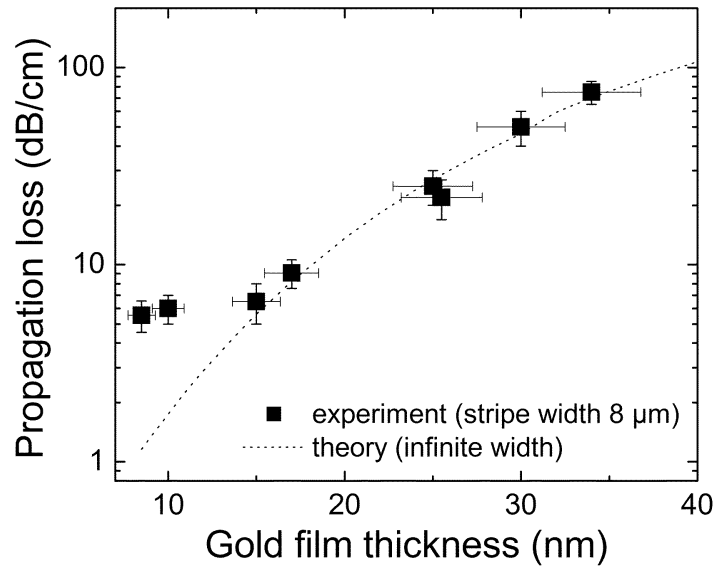

Fig. 6. Experimental measurements of the propagation loss dependence on the thickness of the $8-\mu \mathrm{m}$-wide stripe at the wavelength of $1550 \mathrm{~nm}$, together with the propagation loss curve calculated for infinitely wide stripes.

the propagation loss in practice due to fabrication difficulties in creating a very thin homogeneous metal layer. Since the flatness of a nanometer-thin film can be strongly influenced by that of a substrate surface, it is a rough polymer surface that sets, in our case, a 10-15-nm limit on the thickness of a film exhibiting thickness variations on the scale much smaller that the thickness itself.

In order to study the LR-SPP mode profile, the output intensity distribution from a stripe waveguide was monitored with a microscope arrangement imaging the waveguide output on an infrared Vidicon camera. The output intensity distribution at the output of the 15-nm-thick stripe waveguide for three different waveguide widths $(4,8$, and $12 \mu \mathrm{m})$ is shown in Fig. 7. The mode depth profile consists of two exponential decays with the decaying parameters, which are primarily determined by the metal thickness. However, for narrow stripes (less than 6- $\mu \mathrm{m}$-wide), the depth MFD is expected to increase compared with the infinitely wide stripe of the same thickness [27], which is also seen from the experimentally obtained mode profiles (Fig. 7). The LR-SPP depth profile for an $8-\mu \mathrm{m}$-wide stripe together with the exponential fits is presented in Fig. 8, showing quite a good match except for around the zero-depth coordinate, where the intensity distribution was smoothened to Gaussian-like shape due to the limited resolution $(1-1.5 \mu \mathrm{m})$ of the imaging system.

The lateral mode-field profile was found to fit perfectly to a Gaussian distribution (see inset in Fig. 4). The lateral MFD determined as a function of the 15-nm-thick stripe width (from $2-12 \mu \mathrm{m})$ is presented in Fig. 4. It is seen that the lateral MFD decreases from $\sim 12 \mu \mathrm{m}$ for the stripe width of $12 \mu \mathrm{m}$ to $\sim 10 \mu \mathrm{m}$ for $6-8-\mu \mathrm{m}$-wide stripes, following the decrease in the waveguide width, and then starts to increase, reaching $\sim 16 \mu \mathrm{m}$ for a $2-\mu \mathrm{m}$-wide stripe waveguide, due to weaker light confinement for narrow stripes [27]. This behavior is found to be in good agreement with the results of our simulations (Fig. 4).

The described features of the LR-SPP mode profile in lateral and transverse directions provide the possibility to significantly reduce the coupling loss between an LR-SPP stripe waveguide 

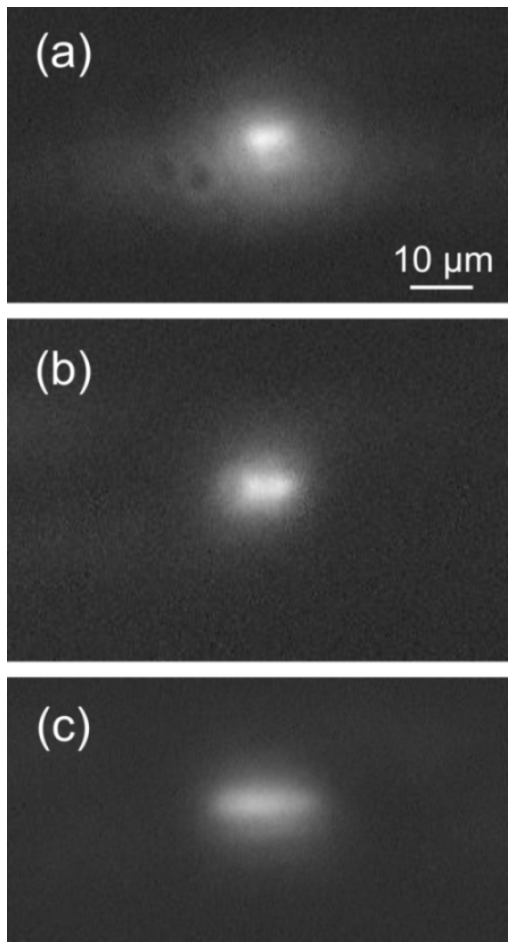

Fig. 7. Output intensity distribution at the output of the 15-nm-thick stripe waveguide for a (a) 4-, (b) 8-, and (c) 12- $\mu$ m-wide (c) stripe.

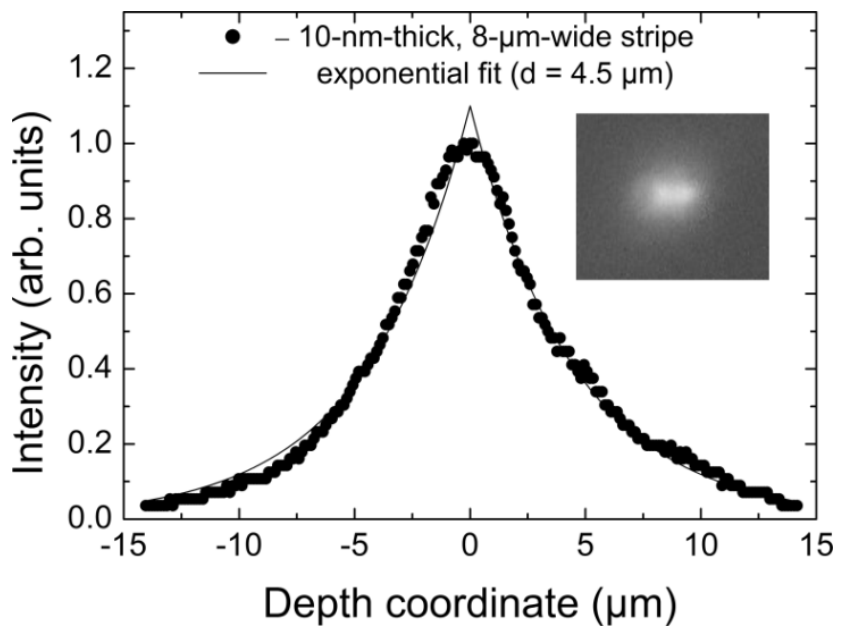

Fig. 8. LR-SPP depth profile for an $8-\mu \mathrm{m}$-wide stripe, together with the exponential fits. The inset shows an example of the LR-SPP stripe output intensity distribution.

and a standard SMF (down to $\sim 0.1 \mathrm{~dB}$ ) by choosing proper stripe dimensions and thus fitting the LR-SPP mode profile to that of the fiber.

\section{S-Bends}

The connection between two straight waveguides, which are offset with respect to each other, is usually called an S-bend. Several approaches for the design of the S-bends have been reported [22], [23], aiming at low additional loss and moderate increase in device length. An intuitive bend design as a combination of two circular arcs performs already better than the one composed of straight sections with sharp corners (sharp bends), but further improvements are still possible. These are

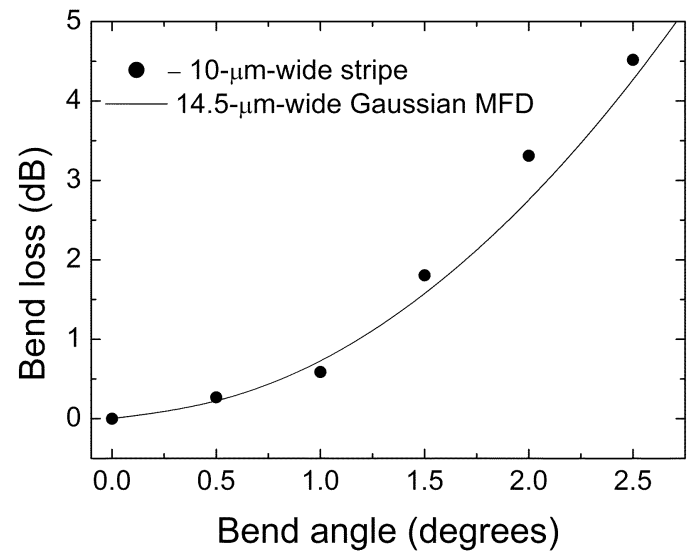

Fig. 9. Bend loss as a function of the bend angle measured for a $10-\mu \mathrm{m}$-wide and 15-nm-thick waveguide in comparison with the quadratic bend loss curve (2) obtained for a Gaussian mode with MFD of $14.5 \mu \mathrm{m}$.

achieved by removing discontinuities not only in the first but also in the second derivative of the bend function, thereby eliminating abrupt curvature changes at the straight-curved transitions and at the midpoint of the S-bend. This approach can be realized with simple designs based on sine and cosine curves.

In our work, we characterized sharp and cosine S-bends based on 10- and 8- $\mu \mathrm{m}$-wide and 15-nm-thick stripe waveguides. The $\mathrm{S}$-bends with the length from 2.5 to $10 \mathrm{~mm}$ were used to connect two straight waveguides with the offset of $200 \mu \mathrm{m}$.

The bend loss for a sharp bend as a function of the bend angle was obtained by normalizing the total insertion loss through the bend to the total loss for the straight waveguide of the same length (Fig. 9). All measurements were performed at the wavelength of $1550 \mathrm{~nm}$. We estimated the bend loss using the simple expression [29]

$$
\text { Loss } \cong 4.343\left(\frac{\pi n_{\mathrm{cl}} W \theta}{2 \lambda}\right)^{2}
$$

and obtained good agreement with the experimental results.

The total insertion loss through a 13-mm-long sample, which contained straight waveguides and S-bends, as a function of curvature radius is shown in Fig. 10, together with the calculated curve [23]. The flat plateau starting from bend radius of approximately $40 \mathrm{~mm}$ gives the low limit for the bend radius of a lossless bend. The bends contribute with the additional loss of $\sim 2$ $\mathrm{dB}$ when the bend radius is about $20-25 \mathrm{~mm}$ (corresponding to the $200-\mu \mathrm{m}$-offset $\mathrm{S}$-bend length of $4.5 \mathrm{~mm}$ ). For S-bend lengths shorter than $4 \mathrm{~mm}$ (bend radius smaller than $16 \mathrm{~mm}$ ), the bend loss increases drastically, making these bends unsuitable for practical applications.

Preliminary investigations showed that further (minor) improvements of the S-bend performance seem possible by choosing other $\mathrm{S}$-bend types, for example, $\sin x-x$ bend, which give slightly lower bend loss for the same waveguide offset and bend length. Some reduction in the bend loss can also be achieved by optimizing the stripe dimensions so as to increase the lateral mode confinement while keeping low the LR-SPP propagation loss (in general, the propagation loss is larger for LR-SPP modes with better confinement). 


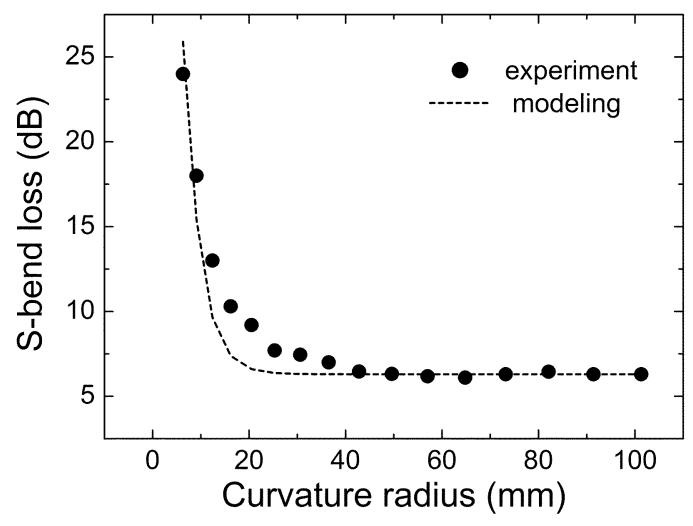

Fig. 10. Total insertion loss through a 13-mm-long sample containing S-bends as a function of curvature radius along with the calculated curve [23].

\section{E. Y-Splitters}

Since single-mode Y-branch waveguides are key elements in the realization of power dividers, optical switches, and Mach-Zehnder modulators, Y-splitters were also included in our mask design and optically characterized in the $S, C$, and $L$ band. We investigated Y-branches, consisting of 15-nm-thick and $8-\mu \mathrm{m}$-wide LR-SPP stripe waveguides, with an arms separation of $250 \mu \mathrm{m}$, which corresponds to the standard pitch of a fiber array, and different splitting lengths from 5-10 $\mathrm{mm}$. Typical transmission spectra for both arms of the Y-splitters, together with the transmission through a straight waveguide of the same length, are shown in Fig. 11(a). For the straight waveguide, the total insertion loss of about 5.5-7 dB in the wavelength range from $1475-1610 \mathrm{~nm}$ includes the propagation loss over $13 \mathrm{~mm}$ and two coupling losses (coupling to the input and output fibers), while the shape of the curve is due to the absorption curve of the polymer. By normalizing the transmission through the $\mathrm{Y}$-splitter to the transmission through the straight waveguide, the loss induced by the Y-splitter was estimated for each arm of the Y-branch. For the splitting length of $10 \mathrm{~mm}$, we demonstrated an essentially lossless 3-dB Y-splitter, while a 5-mm-long Y-splitter was found to induce additional 0.5-dB loss for each arm [Fig. 11(b)]. The difference in the transmission level for the two arms of the investigated Y-splitters was found to be within $0.3 \mathrm{~dB}$, implying that such a device can be used in practice as a symmetrical power divider or $3-\mathrm{dB}$ coupler.

\section{F. MMI Devices}

MMI-based devices that have become important components within integrated optical circuits can also be realized with the LR-SPP-based technology. To demonstrate an LR-SPP-based MMI that performs power splitting, we fabricated 40- and 60- $\mu \mathrm{m}$-wide LR-SPP stripe waveguides (waveguide thickness is $15 \mathrm{~nm}$ ) that were cut into samples of different length. The width of the LR-SPP waveguides was chosen so that the waveguides were multimoded, which in our case of 15 -nm-thick waveguides corresponds to a stripe width of more than $12 \mu \mathrm{m}$ (the cutoff width for the second mode).

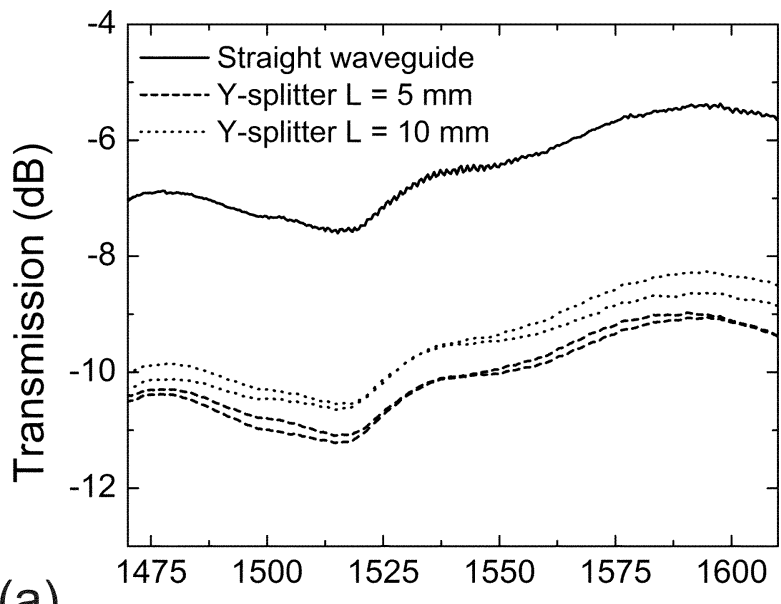

(a)

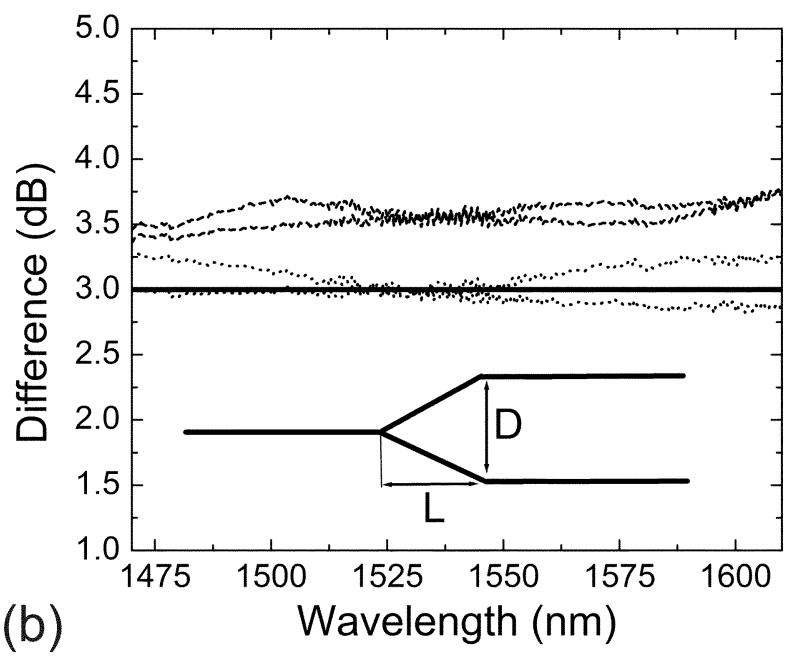

Fig. 11. (a)Transmission spectra for both arms of the 5- and 10-mm-long Y-splitters with $250-\mu \mathrm{m}$ arm separation, together with the transmission through a straight waveguide of the same length; (b) relative transmission spectra.

We analyzed the experimentally obtained output intensity distributions for MMIs of different widths and lengths (Figs. 12 and 13) and compared these with the intensity distribution patterns expected from the modeling. The beat length between the two lowest order modes $L_{\pi}$ can be expressed as (a description of the self-imaging principle as well as a complete analysis can be found in [26])

$$
L_{\pi}=\frac{\pi}{\left(\beta_{0}-\beta_{1}\right)}=\frac{\lambda}{2\left(N_{0}^{\text {eff }}-N_{1}^{\text {eff }}\right)}
$$

where $\lambda$ is the wavelength, and $\beta_{0}, \beta_{1}, N_{0}^{\text {eff }}$, and $N_{1}^{\text {eff }}$ are propagation constants and effective indexes of the zero- and first-order mode, respectively, calculated by using the effective-index approach as before. For an injection (single-mode) waveguide positioned symmetrically with respect to the MMI center line, $N$-fold images are obtained at distances [26]

$$
L_{\pi}=\frac{p}{N}\left(\frac{3 L_{\pi}}{4}\right) \text { with } p=0,1,2, \ldots
$$

For a $40-\mu \mathrm{m}$-wide waveguide, the distance from the input plane to the twofold image plane was estimated to be $3.49 \mathrm{~mm}$, which agrees very well with the output intensity distribution of 

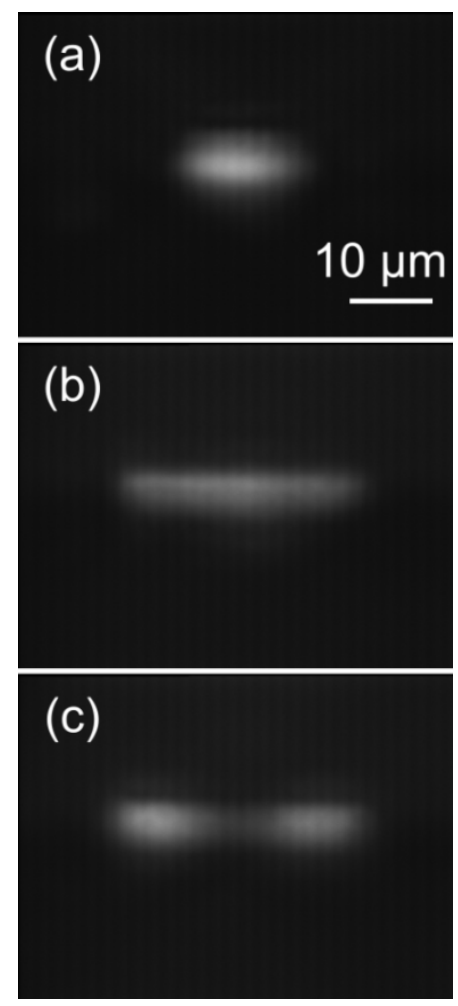

Fig. 12. Output intensity distributions for $40-\mu \mathrm{m}$-wide MMIs of different lengths: (a) 2.3-mm-long - the value for distance to onefold image ((3), (4)) expected from the modeling is $2.33 \mathrm{~mm}$; (b) 2.8 - $\mathrm{mm}$-long; and (c) $3.3-\mathrm{mm}$-long (distance to twofold image from modeling is $3.49 \mathrm{~mm}$ ).

the $40-\mu \mathrm{m}$-wide waveguide of $3.3 \mathrm{~mm}$ length. Experimentally observed fourfold image for the $60-\mu \mathrm{m}$-wide and 5.5-mm-long waveguide was also found to be in excellent agreement with the simulations that predicted the fourfold image plane to be 5.59 $\mathrm{mm}$ from the input plane.

\section{G. Directional Couplers}

We investigated DCs based on 8 - $\mu \mathrm{m}$-wide stripe waveguides with different separation distances between waveguides (edge-to-edge) (Fig. 14). DCs with an interaction length $L$ varying from 0.1 to $1.5 \mathrm{~mm}$ in $0.1-\mathrm{mm}$ steps, were investigated. For spectrally dependent measurements, devices with large interaction length $(L=8 \mathrm{~mm})$ were used.

Power transmitted through the direct $I_{I I}$ and coupled $I_{X}$ arms of a DC can be written as

$$
\begin{aligned}
I_{I I} & =\cos ^{2}(\kappa L) \\
I_{X} & =\sin ^{2}(\kappa L)
\end{aligned}
$$

where $\kappa \propto(1 / \lambda)$ is a wavelength-dependent coupling constant. The coupling length corresponding to full power transfer from one arm to another is then defined as

$$
L_{\frac{\pi}{2}}=\frac{\pi}{2 \kappa} \text {. }
$$

Typical transmission spectra for the direct and coupled arms of the DC based on $8-\mu \mathrm{m}$-wide waveguides with $6-\mu \mathrm{m}$ separation (interaction length of $8 \mathrm{~mm}$ ) are presented in Fig. 15, showing efficient power transfer from one arm to another and proving the operational principle of a DC. An example of output
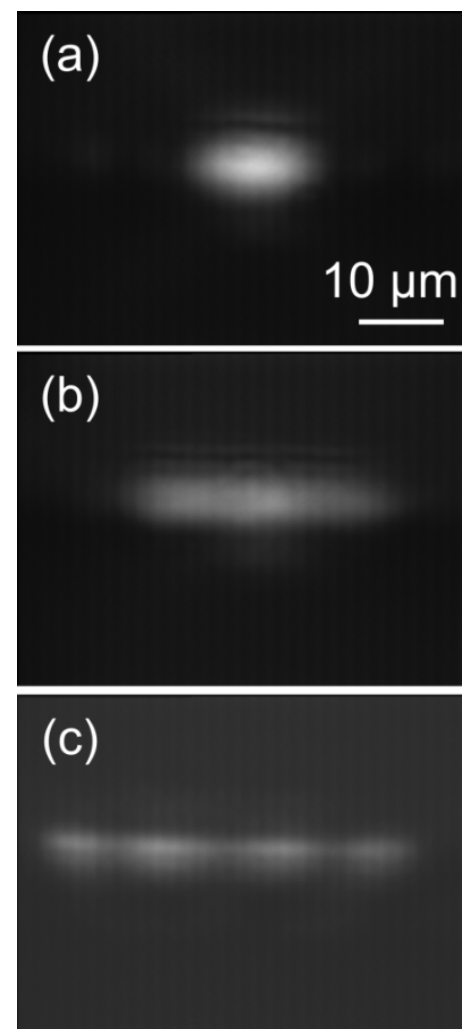

Fig. 13. Output intensity distributions for $60-\mu \mathrm{m}$-wide MMIs of different lengths: (a) 4.6-mm-long-the value for distance to onefold image ((3), (4)) expected from the modeling is $2.47 \mathrm{~mm}$; (b) 5.0-mm-long; and (c) 5.5-mm-long (distance to twofold image from modeling is $5.59 \mathrm{~mm}$ ).

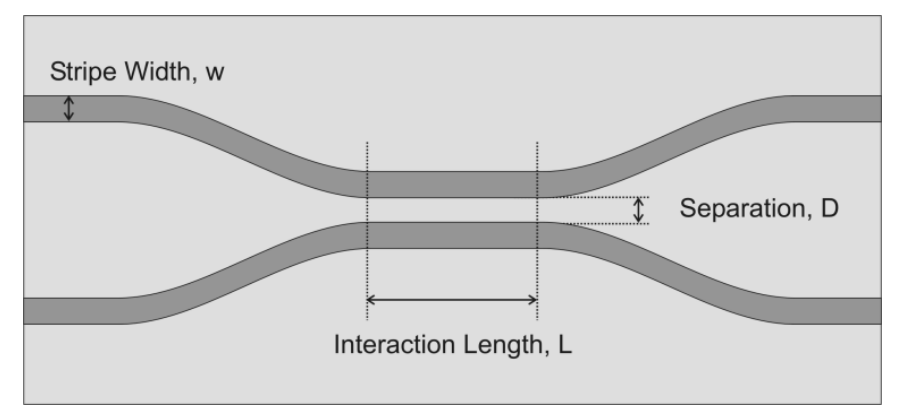

Fig. 14. Schematic of the DC constructed from straight waveguides and S-bends.

modes from two DC arms is shown in the inset of Fig. 16(a) for a waveguide width $8 \mu \mathrm{m}$ and separation $4 \mu \mathrm{m}$, demonstrating a well-defined output mode from both arms and low level of background light in the polymer cladding.

For DCs with the interaction length changing from 0.1 to 1.5 $\mathrm{mm}$ with a $0.1-\mathrm{mm}$ step, the transmission through the direct and coupled arm was measured at the wavelength of $1570 \mathrm{~nm}$ using a tunable laser. The dependence of the transmitted power through one of the arms on interaction length was fitted using the (5) and (6) (Fig. 16) enabling an estimation of the value of the coupling length and the offset, which gives a correction to the nominal interaction length due to the interaction of the modes in the S-bend region. For $8-\mu \mathrm{m}$-wide waveguides, the coupling length $L_{\pi / 2}$ was estimated to be around 0.8 and $1.9 \mathrm{~mm}$ for the separation distances of 0 and $4 \mu \mathrm{m}$, respectively. 


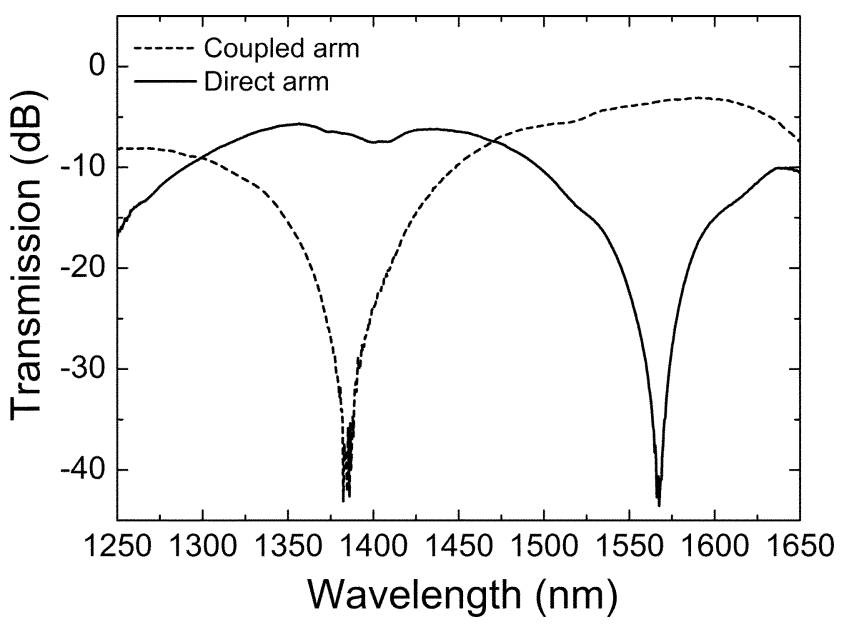

Fig. 15. Typical transmission spectra for the direct and coupled arms of the DC based on $8-\mu \mathrm{m}$-wide waveguides with the separation of $6 \mu \mathrm{m}$ and interaction length of $8 \mathrm{~mm}$.

Using the previously mentioned effective-refractive-index approach, we estimated the coupling length for a DC with $0-\mu \mathrm{m}$-separated waveguides by calculating the beat length between the first two (lowest order) modes ((3)) of the composite waveguide structure. For a 15 -nm-thick and $16-\mu \mathrm{m}$-wide waveguide, which corresponds to the DC based on $8-\mu \mathrm{m}$-wide stripes with $0-\mu \mathrm{m}$ separation, the beat length was calculated to be $0.91 \mathrm{~mm}$, which is close to the experimentally obtained value of $0.8 \mathrm{~mm}$. For the first two modes of the structure consisting of two $8-\mu \mathrm{m}$-wide waveguides with $4-\mu \mathrm{m}$ separation, the beat (coupling) length was found to be $1.93 \mathrm{~mm}$, matching precisely the experimental value of $1.9 \mathrm{~mm}$ for the DC with $4-\mu \mathrm{m}$-separated waveguides.

The offset value shows that the interaction between two waveguides starts earlier than the parallel section. For the case of $0-\mu \mathrm{m}$ separation between waveguides, the offset value obtained from the $\sin ^{2}$ fit was $1.3 \mathrm{~mm}$ [Fig. 16(a)], while for the $8-\mu \mathrm{m}$-separated waveguides, this distance was about 0.7 $\mathrm{mm}$ [Fig. 16(b)]. These DCs were composed of 2.5-mm-long cosine-bends with the offset of $48 \mu \mathrm{m}$. The results for the offsets showed that the interaction of the modes started in the area of the S-bend, when the distance between waveguide centers was approximately twice as large as that in the nominal interaction region. This has to be taken into account when designing waveguide components containing DC devices.

\section{CONCLUSION}

Polymer-based LR-SPP stripe waveguide components constitute a new alternative for integrated optical circuits. In this paper, the guiding and routing of light along thin gold stripes embedded in polymer via excitation of LR-SPPs were investigated and LR-SPP properties such as MFD, propagation, and coupling loss at the telecom wavelengths were studied. The propagation loss of $\sim 6 \mathrm{~dB} / \mathrm{cm}$ and coupling loss of $\sim 0.5 \mathrm{~dB}$ (per facet) have been obtained for 15 -nm-thick stripe waveguides of $8 \mu \mathrm{m}$ width. The performance of the S-bends with the offset of $200 \mu \mathrm{m}$ has been studied, and the S-bend loss of less than $3 \mathrm{~dB}$ has been obtained for a 4.5 -mm-long S-bend. A lossless Y-splitter that can be used as a symmetrical power
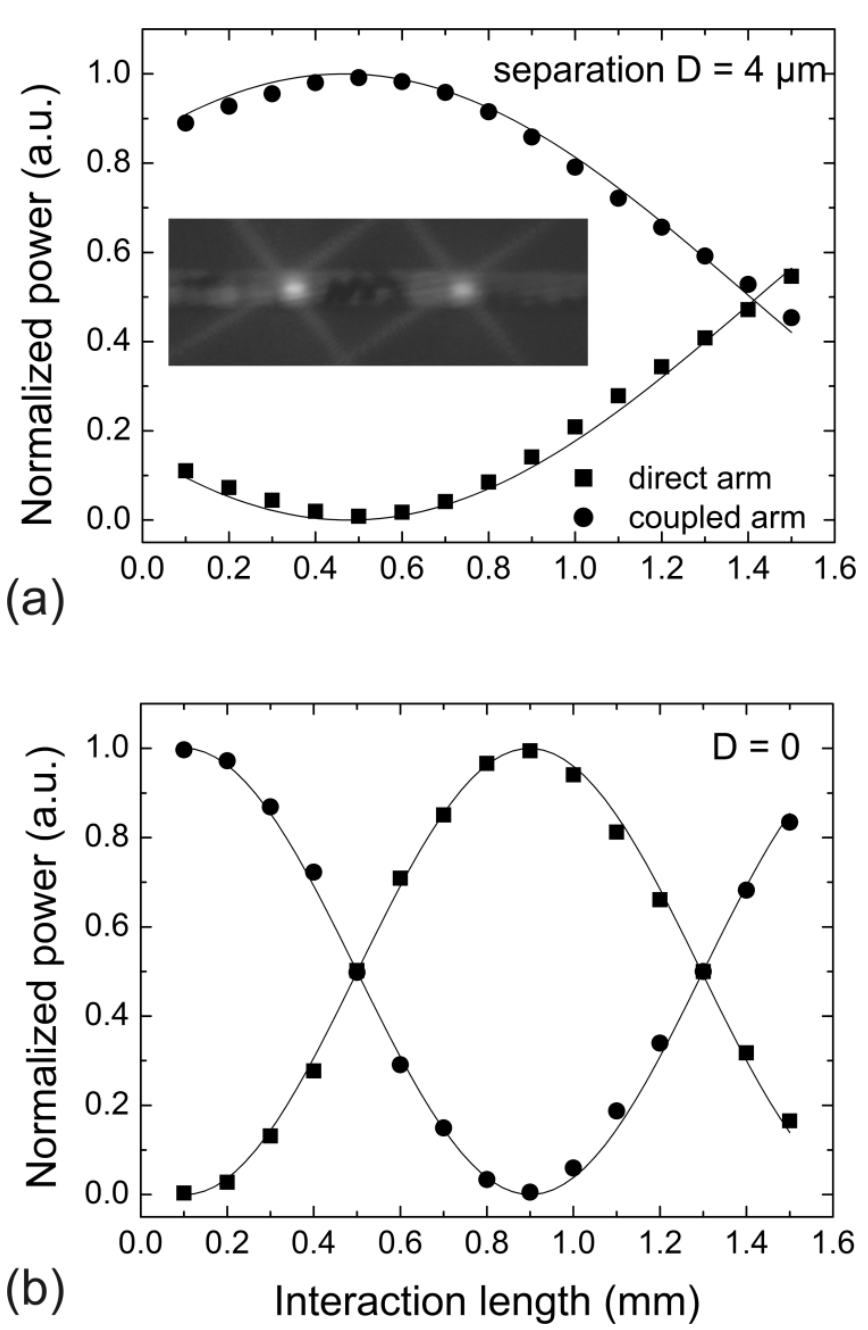

Fig. 16. Dependence of the transmitted power through the direct and coupled arms on the interaction length for DCs based on 8- $\mu \mathrm{m}$-wide waveguides with (a) 4- and $0-\mu \mathrm{m}$ separation (b) between waveguides together with the $\sin ^{2}$ and $\cos ^{2}$ fits ((5), (6)) giving the coupling length of 1.9 and $0.8 \mathrm{~mm}$, respectively. The inset shows an example of the DC output for the waveguide widths $8 \mu \mathrm{m}$ and separation $4 \mu \mathrm{m}$. The output arms separation is $112 \mu \mathrm{m}$.

divider or 3-dB coupler has been demonstrated. MMI devices based on LR-SPP stripe waveguides have been demonstrated opening up the possibility of designing different routing and coupling components as well as wavelength demultiplexing elements based on LR-SPP MMIs. DCs based on 15-nm-thick and 8 - $\mu \mathrm{m}$-wide stripe waveguides have also been investigated, and coupling lengths of 1.9 and $0.8 \mathrm{~mm}$ have been found for DCs with 4- and 0- $\mu \mathrm{m}$-separated waveguides, respectively, implying that quite short (a few millimeters) and thereby low-loss functional components (splitters, couplers, etc.) can be realized. All LR-SPP waveguide components have been modeled using the effective-refractive-index approach, and good agreement with the experimental results has been obtained.

LR-SPP waveguide devices have emerged as a new class of components that can provide guiding and routing of light as well as coupling and splitting from/into a number of channels with reasonable insertion loss and good balancing. Since all conventional passive circuits can be fabricated on the base of LR-SPP stripe waveguides, some advantages of LR-SPP-technology are worth mentioning here. In the first place, it is truly 
planar technology (the metal thickness is negligibly small) based on a simple and low-cost fabrication procedure involving the standard photolithography and metallization processes. Second, the efficient coupling with SMF (ideally as low as 0.1 $\mathrm{dB})$ is achievable due to the possibility of the mode shaping by choosing proper waveguide dimensions. The propagation loss of $\sim 6 \mathrm{~dB} / \mathrm{cm}$ can, in principle, be reduced down to at least $1.5 \mathrm{~dB} / \mathrm{cm}$ via material and processing optimization. The design procedure for LR-SPP waveguide components can be performed quite easily using a numerical mode solver and the effective-index approach. Finally, the very same metal stripes that are used for the radiation guiding can be employed as electrodes carrying signal currents influencing the waveguiding characteristics, e.g., thermo-, electro- or magnetooptic effects, depending on the cladding material. Further investigations in this area are being conducted and increasing exploitation of the LR-SPP waveguide components is foreseen in the area of integrated optics.

\section{REFERENCES}

[1] Y. P. Li and C. H. Henry, "Silica-based optical integrated circuits," Proc. Inst. Elect. Eng. Optoelectronics, vol. 143, pp. 263-280, 1996.

[2] R. M. d. Ridder, K. Worhoff, A. Driessen, P. V. Lambeck, and H. Albers, "Silicon oxynitride planar waveguiding structures for applications in optical communication," IEEE J. Sel. Topics Quantum Electron., vol. 4, no. 6, pp. 930-937, Nov.-Dec. 1998.

[3] M. F. Grant, "Integrated optical waveguide devices on silicon for optical communications," in IEE Colloquium 'Planar Silicon Hybrid Optoelectronics Dig. 1994/198, 1994, p. 1-1-1/10.

[4] R. Chakraborty, "Integrated optical waveguides in $\mathrm{LiNbO}_{3}$ : Modeling and experimental analysis," in Proc. SPIE-Int. Soc. Optical Engineering, vol. 4417, 2001, pp. 278-285.

[5] R. M. Almeida, "Sol-gel planar waveguides for integrated optics," $J$. Non-Cryst. Solids, vol. 259, pp. 176-181, 1999.

[6] L. A. Eldada, "Polymer integrated optics: Promise versus practicality," in Proc. SPIE-Int. Soc. Optical Engineering, 2002, pp. 11-22.

[7] P. Coudray, P. Etienne, and Y. Moreau, "Integrated optics based on organo-mineral materials," Material Science Semiconductor Processing, vol. 3, pp. 331-337, 2000.

[8] H. Raether, Surface Plasmons. Berlin, Germany: Springer-Verlag, 1998.

[9] W. L. Barnes, A. Dereux, and T. W. Ebbesen, "Surface plasmon subwavelength optics," Nature, vol. 424, pp. 824-830, 2003.

[10] B. Hecht, H. Bielefeld, L. Novotny, Y. Inouye, and D. W. Pohl, "Local excitation, scattering, and interference of surface plasmons," Phys. Rev. Lett., vol. 77, pp. 1889-1892, 1996.

[11] S. I. Bozhevolnyi and F. A. Pudonin, "Two-dimensional micro-optics of surface plasmons," Phys. Rev. Lett., vol. 78, pp. 2823-2826, 1997.

[12] B. Lamprecht, J. R. Krenn, G. Schider, H. Ditlbacher, M. Salerno, N. Felidj, A. Leitner, F. R. Aussenegg, and J. C. Weeber, "Surface plasmon propagation in microscale metal stripes," Appl. Phys. Lett., vol. 79, pp. 51-53, 2001.

[13] S. I. Bozhevolnyi, J. Erland, K. Leosson, P. M. W. Skovgaard, and J. M. Hvam, "Waveguiding in surface plasmon polariton band gap structures," Phys. Rev. Lett., vol. 86, pp. 3008-3011, 2001.

[14] S. I. Bozhevolnyi, V. S. Volkov, K. Leosson, and A. Boltasseva, "Bend loss in surface plasmon polariton band-gap structures," Appl. Phys. Lett., vol. 79, pp. 1076-1078, 2001.

[15] S. I. Bozhevolnyi, V. S. Volkov, K. Leosson, and J. Erland, "Observation of propagation of surface plasmon polaritons along line defects in a periodically corrugated metal surface," Opt. Lett., vol. 26, pp. 734-736, 2001.

[16] J.-C. Weeber, A. Dereux, and C. Girard, "Plasmon polaritons of metallic nanowires for controlling submicron propagation of light," Phys. Rev. B, Condens. Matter, vol. 60, pp. 9061-9068, 1999.
[17] J.-C. Weeber, J. R. Krenn, A. Dereux, B. Lamprecht, Y. Lacroute, and J.-P. Goudonnet, "Near-field observation of surface plasmon polariton propagation on thin metal stripes," Phys. Rev. B, Condens. Matter, vol. 64, pp. 045 411-045 419, 2001.

[18] D. Sarid, "Long-range surface-plasma waves on very thin metal films," Phys. Rev. Lett., vol. 47, pp. 1927-1930, 1981.

[19] J. J. Burke, G. I. Stegeman, and T. Tamir, "Surface-polariton-like waves guided by thin, lossy metal films," Phys. Rev. B, Condens. Matter, vol. 33, pp. 5186-5201, 1986.

[20] R. Charbonneau, P. Berini, E. Berolo, and E. Lisicka-Skrzek, "Experimental observation of plasmon-polariton waves supported by a thin metal film of finite width," Opt. Lett., vol. 25, pp. 844-846, 2000.

[21] T. Nikolajsen, K. Leosson, I. Salakhutdinov, and S. I. Bozhevolnyi, "Polymer-based surface-plasmon-polariton stripe waveguides at telecommunication wavelengths," Appl. Phys. Lett., vol. 82, pp. 668-670, 2003.

[22] Y. Y. Lu and P. L. Ho, "Beam propagation modeling of arbitrarily bent waveguides," IEEE Photon. Technol. Lett., vol. 14, no. 12, pp. 1698-1700, Dec. 2002.

[23] A. Kumar and S. Aditya, "Performance of S-bends for integrated-optic waveguides," Microwave Optical Technology Lett., vol. 19, pp. 289-292, 1998.

[24] H. v. Brug, F. H. Groen, Y. S. Oei, J. W. Pedersen, E. C. M. Pennings, D. K. Doeksen, and J. J. G. M. v. d. Tol, "Low-loss straight and curved ridge waveguides in LPE-grown GaInAsP," Electron. Lett., vol. 25, pp. 1330-1332, 1989.

[25] R. C. Alferness, "Guided-wave devices for optical communication," IEEE J. Quantum Electron., vol. QE-17, no. 6, pp. 946-959, Jun. 1981.

[26] L. B. Soldano and E. C. M. Pennings, "Optical multi-mode interference devices based on self-imaging: Principles and applications," J. Lightw. Technol., vol. 13, no. 4, pp. 615-627, Apr. 1995.

[27] P. Berini, "Plasmon-polariton waves guided by thin lossy metal films of finite width: bound modes of symmetric structures," Phys. Rev. B, Condens. Matter, vol. 61, pp. 10 484-10 503, 2000.

[28] H. Kogelnik, Theory of Dielectric Waveguides, T. Tamir, Ed. Berlin, Germany: Springer-Verlag, 1979, Topics in Applied Physics, pp. 64-66.

Alexandra Boltasseva, photograph and biography not available at the time of publication.

Thomas Nikolajsen, photograph and biography not available at the time of publication.

Kristjan Leosson, photograph and biography not available at the time of publication.

Kasper Kjaer, photograph and biography not available at the time of publication.

Morten S. Larsen, photograph and biography not available at the time of publication.

Sergey I. Bozhevolnyi, photograph and biography not available at the time of publication. 